\title{
An efficient Scheme to Remove Low Density Impulse Noise From A
}

\author{
Digital Image \\ Jihad N. Abdeljaliil ${ }^{1}$ \\ BY DR Jihad N. Abdeljalil Al-Balqa' Applied University/ Faculty of Engineering \\ Technology,Amman, Jordan. \\ Email: jihadnader@yahoo.com
}

\begin{abstract}
An improved adaptive noise reduction scheme for images that are highly corrupted by Salt-and-Pepper noise(impulse noise), is proposed in this paper which efficiently removes the salt and pepper noise while preserving the details. The proposed scheme efficiently identifies and reduces salt and pepper noise. The algorithm utilizes an IIR filter with controlled parameters to get better image quality than the existing methods of noise removing. A comparative analysis between the proposed scheme and other techniques of noise reduction or removing is presented in order to show the advantages of the proposed scheme in removing the noisy pixels and producing a better PSNR.
\end{abstract}

\section{KEYWORDS}

Impulse Noise, Noisy Image, MSE, PSNR, IIR, FIR

\section{INTRODUCTION}

The two most common types of noise in digital image processing are Gaussian and Impulse noises, also known as salt and pepper noise. This type of noise is a special type of noise which can have many different origins. Images are often corrupted by impulse noise caused by transmission errors, faulty memory locations or timing errors in analog-to-digital conversion. Salt and-pepper noise is one type of impulse noise which can corrupt the image, where the noisy pixels can take only the maximum and minimum values in the dynamic range. Since, linear filtering techniques are not effective in removing impulse noise, non-linear filtering techniques are widely used in the restoration process. The standard median filter (SMF) is one of the most popular non-linear filters used to remove salt and-pepper noise due to its good demising power and computational efficiency [3]. However, the major drawback of the SMF is that, the filter is effective only for low noise densities, and additionally, exhibits blurring if the window size is large and leads to insufficient noise suppression if the window size is small [4]. When the noise level is over $50 \%$, the edge details of the original image will not be preserved by the median filter [2]. Nevertheless, it is important that during

It is important that during the filtering (restoration) process the edge details have to be preserved without losing the high frequency components of the image edges [4], [5].

The ideal approach is to apply the filtering technique only to noisy pixels, without changing the uncorrupted pixel values. Non-linear filters such as Adaptive Median Filter (AMF), decision-based or switching median filters [6], [7], [8] can be used for discriminating corrupted and uncorrupted pixels, and then apply the filtering technique. Noisy pixels will be replaced by the median value and uncorrupted pixels will be left unchanged. AMF performs well at low noise densities since the corrupted pixels which are replaced by the median values are very few. At higher noise densities, window size has to be increased to get better noise removal which will lead to less correlation between corrupted pixel values and replaced median pixel values. In decision-based or switching median filter the decision is based on a pre-defined threshold value. The major drawback of this method is that defining a robust decision measure is difficult. Also these filters will not take into account the local features as a result of which details and edges may not be recovered satisfactorily, especially when the noise level is high. Chan et al., [2] proposed an algorithm to overcome this problem, which consists of two stages. The first stage is to classify the corrupted and uncorrupted pixels by using AMF and in the second stage, regularization method is applied to the corrupted pixels to preserve edges and suppress noise. The drawback of this method is that for high impulse noise, it requires large window size of $39 \times 39$, and additionally requires complex circuitry for the implementation and determination of smoothing factor $\beta$ to get good results [2]. Srinivasan and Ebenezer [1] proposed an algorithm in which the corrupted pixels are replaced by either the median pixel or neighborhood pixel by using a fixed window size of $3 \times 3$ resulting in lower processing time and good edge preservation. Although the recent technique [1] showed promising results, we discovered that a smooth transition between the pixels is lost leading to degradation in the visual quality of the image, since it only considers the left neighborhood from the last processed value. To overcome this problem we propose a new algorithm in this paper, where corrupted pixels can either be replaced by the median pixel or, the mean of the neighborhood processed pixels, which results in a smooth transition between the pixels with edge preservation and better visual quality. In addition, may appear in digital image due contaminated impulse noise, which is caused by malfunctioning pixels in camera sensors, faulty memory location in hardware, or transmission in noisy channel [8]. Salt and pepper noise scattered throughout the image in such a way pixels can take only the maximum and minimum values $(0$ and 255 respectively) in the dynamic range. 
Madhu S. Nair, K. Revathy, and Rao Tatavarti [9] proposed an efficient algorithm for removing impulse noise and it was compared with other algorithms in order to show the advantages of the proposed algorithm, this algorithm regardless it's advantages gave a lower Peak signal-to-noise ratio (PSNR).

\section{PEAK SIGNAL -TO-NOISE RATIO}

Peak signal-to-noise ratio( PSNR), is an engineering term for the ratio between the maximum possible power of a signal and the power of corrupting noise that affects the fidelity of its representation. Because many signals have a very wide dynamic range, PSNR is usually expressed in terms of the logarithmic decibel scale.

PSNR is most commonly used to measure the quality of reconstruction of loss compression codec's (e.g., for image compression). The signal in this case is the original data, and the noise is the error introduced by compression. When comparing compression codec's, PSNR is an approximation to human perception of reconstruction quality. Although a higher PSNR (see figure 1) generally indicates that the reconstruction is of higher quality, in some cases it may not. One has to be extremely careful with the range of validity of this metric; it is only conclusively valid when it is used to compare results from the same codec (or codec type) and same content.[1][2]

PSNR is most easily defined via the mean squared error (MSE)[10],[11], and[12]. Given a noise-free m $\times n$ monochrome image I and its noisy approximation K, MSE is defined as:

$$
\text { MSE }=\frac{1}{m n} \sum_{i=0}^{m-1} \sum_{j=0}^{n-1}[I(i, j)-K(i, j)]^{2}(1)
$$

The PSNR (in $\mathrm{dB}$ ) is defined as:

$$
\text { PSNR }=10 \cdot \log _{10}\left(\frac{M A X_{1}^{2}}{M S E}\right)(2)
$$

Here, MAXI is the maximum possible pixel value of the image. When the pixels are represented using 8 bits per sample, this is 255.

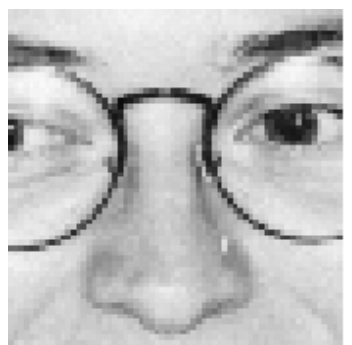

PSNR $=40 \mathrm{~dB}$

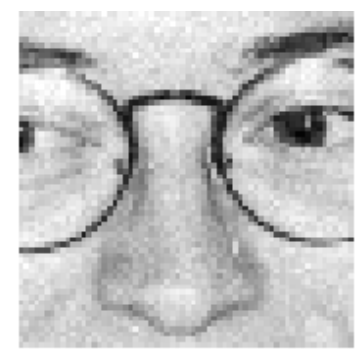

PSNR $=30 \mathrm{~dB}$

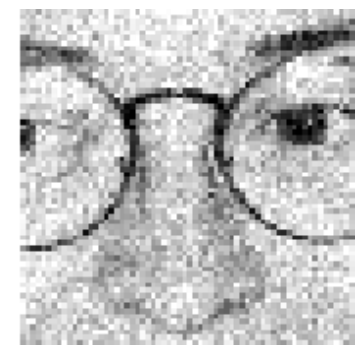

PSNR $=20 \mathrm{~dB}$

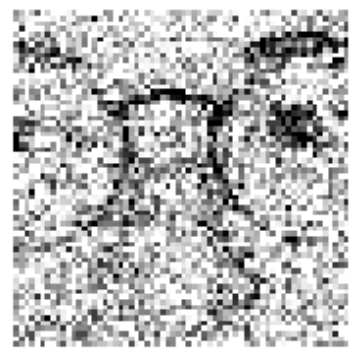

PSNR $=10 \mathrm{~dB}$

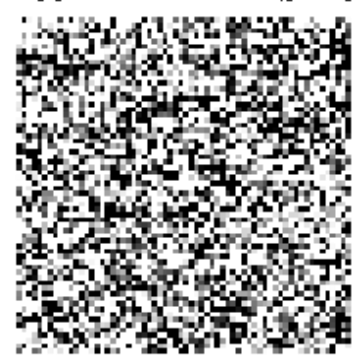

PSNR $=0 \mathrm{~dB}$

Fig. 1. Illustration of the PSNR measure

\section{THE PROPOSED SCHEME}

The proposed scheme uses an infinite impulse response (IIR) or finite impulse response (FIR) filter. The filter function is implemented as a direct form II transposed structure as shown in figure 2, 


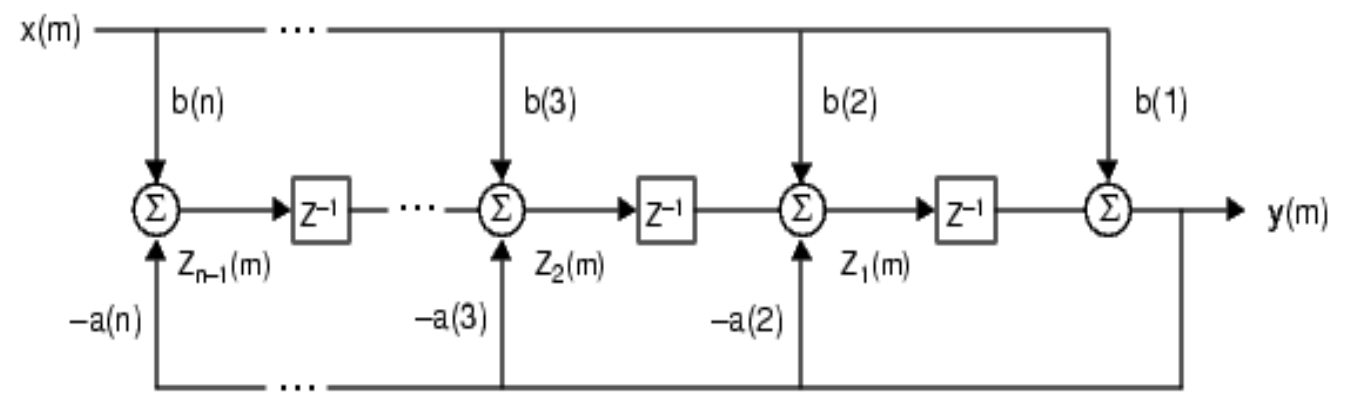

Fig. 2. IIR structure

Or

$y(n)=b(1)^{*} x(n)+b(2)^{*} x(n-1)+\ldots+b(n b+1)^{*} x(n-n b)-a(2)^{*} y(n-1)-\ldots-a(n a+1)^{*} y(n-n a)$

Here $a(2), a(n a+1)$ are the controlled parametors of the digital filter.

The operation of filter at sample $\mathrm{mm}$ is given by the time domain difference equations:

$$
\begin{aligned}
& \mathrm{y}(\mathrm{m})=\mathrm{b}(1) \mathrm{x}(\mathrm{m})+z_{1}(m-1) \\
& z_{1}(m)=\mathrm{b}(2) \mathrm{x}(\mathrm{m})+z_{2}(m-1)-a(2) y(m) \\
& \vdots=\vdots \\
& z_{n-2}(m)=\mathrm{b}(n-1) \mathrm{x}(\mathrm{m})+z_{n-1}(m-1)-a(n-1) y(m) \\
& z_{n-1}(m)=\mathrm{b}(\mathrm{n}) \mathrm{x}(\mathrm{m})-a(n) y(m)
\end{aligned}
$$

The input-output description of this filtering operation in the z-transform domain is a rational transfer function,

$$
\mathrm{Y}(\mathrm{z})=\frac{b(1)+b(2) z^{-1}+\cdots+b(n b+1) z^{-n b}}{1+a(2) z^{-1}+\ldots+a(n a+1) z^{-n a}} \mathrm{X}(\mathrm{z})
$$

To use the filter shown in figure 2 to remove impulse noise we have to apply the following steps:

- Set the IIR filter parameter (we use only a2 in this paper.

- Initialize the numerator of the Z-transform.

- Initialize the denominator of the z-transform.

- Reshape the digital image into one column vector.

- Apply the filter.

- Reshape the output of the filter to 2 dimensional matrix (gray digital image).

\section{IMPLEMENTATION}

Gray-scale images such as cameraman.tif of size $256 \times 256$ has been used to test the performance of the proposed schema with dynamic range of values $(0,255)$. Figure 3 shows the results of image filtering: 


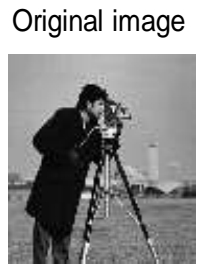

Noisy image

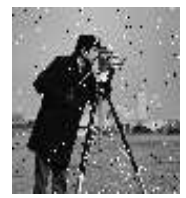

filtered image

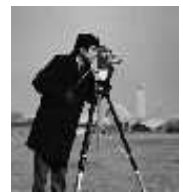

Fig 3: Filtering results

Tables 1, 2 and 3 show the performance results of the various filters, on the gray-scale images cameraman.tif, at low $(30 \%)$, medium $(60 \%)$ and high noise $(90 \%)$ densities, respectively.

TABLE 1. PSNR AND COMPUTATION TIME FOR VARIOUS FILTERS FOR CAMERAMAN.TIF IMAGES (GRAY-SCALE) AT LOW NOISE DENSITY (30\%)

\begin{tabular}{|c|c|c|c|}
\hline Filter & PSNR(db) & $\begin{array}{c}\text { Filtering } \\
\text { time(s) }\end{array}$ & Remarks \\
\hline Median & 22.6922 & 0.04 & \\
\hline Average & 28.6083 & 0.04 & \\
\hline $\begin{array}{c}\text { Proposed in } \\
\{9]\end{array}$ & 30.79 & 1.77 & $\mathrm{~A} 2=-0.3$ \\
\hline $\begin{array}{c}\text { Proposed } \\
\text { scheme }\end{array}$ & 36.4314 & 0.01 & \\
\hline
\end{tabular}

TABLE 2. PSNR AND COMPUTATION TIME FOR VARIOUS FILTERS FOR cameraman.tif IMAGES (GRAY-SCALE) AT medium NOISE DENSITY (60\%)

\begin{tabular}{|c|c|c|c|}
\hline Filter & PSNR(db) & Filtering time(s) & Remarks \\
\hline Median & 31.6209 & 0.04 & \\
\hline Average & 27.8477 & 0.04 & \\
\hline Proposed in $\{9]$ & 24.95 & 1.86 & $\mathrm{~A} 2=-0.6$ \\
\hline Proposed scheme & 45.6958 & 0.01 & \\
\hline
\end{tabular}


TABLE 3. PSNR AND COMPUTATION TIME FOR VARIOUS FILTERS FOR cameraman.tif IMAGES (GRAY-SCALE) AT High NOISE DENSITY (90\%)

\begin{tabular}{|c|c|c|c|}
\hline Filter & PSNR(db) & Filtering time(s) & Remarks \\
\hline Median & 28.2405 & 0.04 & \\
\hline Average & 27.4724 & 0.04 & \\
\hline Proposed in $\{9]$ & 19.2 & 3.37 & $\mathrm{~A} 2=-0.9$ \\
\hline Proposed scheme & 66.2096 & 0.01 & \\
\hline
\end{tabular}

The experimental results shows that the proposed scheme is very efficient in removing low noise density, and here the scheme can remove only the noisy pixels.

Table 4 shows the correlation coefficients between the original image and the de-noised one when applying the proposed scheme to remove noise with deferent low density values.

TABLE 4. CorRELATION BETWEen tHE ORIGINAL IMAGE AND THE FILTERED IMAGE.

\begin{tabular}{|c|c|}
\hline Noise density(\%) & Correlation coefficients \\
\hline 1 & 0.9835 \\
\hline 2 & 0.9831 \\
\hline 3 & 0.9829 \\
\hline 10 & 0.9784 \\
\hline 30 & 0.9290 \\
\hline 60 & 0.5775 \\
\hline 90 & 0.1159 \\
\hline
\end{tabular}

\section{CONCLUSION}

A new scheme for impulse noise removal was proposed which gave better performance in comparison with other existing noise removal algorithms in terms of PSNR, and computational time. The performance of the proposed scheme has been tested at low, medium and high noise densities on gray-scale images. Even at high noise density levels the proposed scheme gave better results in comparison with other existing algorithms. It was shown that the proposed scheme was very efficient when dealing with impulse noise wilt density leers than $30 \%$.

\section{REFERENCES}

1. K. S. Srinivasan, D. Ebenezer, " A New Fast and Efficient DecisionBased Algorithm for Removal of High-Density Impulse Noises," IEEE Signal Processing Papers, Vol. 14, No. 3, pp. 189-192, March 2007.

2. R. H. Chan, Chung-Wa Ho, M. Nikolova, "Salt and Pepper Noise Removal by Median Type Noise Detectors and Detail -Preserving Regularization,” IEEE Transactions on Image Processing, Vol. 14, No.10, pp. 1479-1485, October 2005.

3. T. S. Huang, G. J. Yang, and G. Y. Tang, "Fast two-dimensional median filtering algorithm," IEEE Trans. Acoustics, Speech, Signal Process., Vol. ASSP-1, No. 1, pp. 13-18, Jan. 1979.

4. C. A. Pomalaza-Racz and C. D. Macgillem, "An adaptive non linear edge preserving filter," IEEE Trans. Acoustics, Speech, Signal Process., Vol. ASSP-32, pp. 571-576, Jun. 1984.

5. T. Sun and Y. Neuvo, "Detail-preserving median based filters in image processing," Pattern Recognition. Lett., vol. 15, pp. 341-347, 1994

6. H. Hwang and R. A. Haddad, "Adaptive median filters: New algorithms and results," IEEE Trans. Image Process., Vol. 4, No. 4, pp. 499-502, Apr. 1995.

7. S. Zhang and M. A. Karim, "A new impulse detector for switching median filters," IEEE Signal Process. Lett,. Vol. 9, No. 11, pp. 360-363, Nov. 2002.

8. H.-L. Eng and K.-K. Ma, "Noise adaptive soft-switching median filter," IEEE Trans. Image Process., vol. 10, no. 2, pp. 242-251, Feb. 2001. 
9. Madhu S. Nair, K. Revathy, and Rao Tatavarti, Removal of Salt-and Pepper Noise in Images: A New DecisionBased Algorithm, Proceedings of the International MultiConference of Engineers and Computer Scientists 2008 Vol I IMECS 2008, 19-21 March, 2008, Hong Kong.

10. Rafael C. Gonzalez and Richard E. Woods. Digital Image Processing( Addison-Wesley 1992)

11. Huynh-Thu, Q.; Ghanbari, M. (2008). "Scope of validity of PSNR in image/video quality assessment". Electronics Letters. 44 (13): 800. doi:10.1049/el:20080522.

12. Oriani, Emanuele. "qpsnr: A quick PSNR/SSIM analyzer for Linux". http://qpsnr.youlink.org/. Retrieved 26 August 2016.

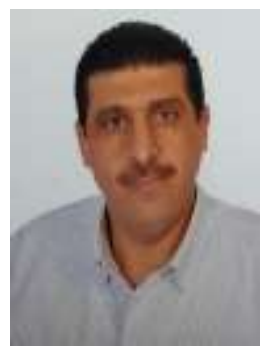

JIHAD N. ABDEL-JALIL received his B.S. degrees in Electronic Computer Complexes \& Networks from Moscow state university of communications means, Russia, in 1994,and the Ph.D. degree in computer engineering from Kursk State Technical University of Kursk, Russia, in 2006. His research interests include image processing, computer networks and routing. 